\title{
The clinial utility of serum leukocyte counts for initial antibiotic use in acute mastitis: the experience from China
}

\author{
Li Jiang ${ }^{1}$, Jianjiang Fang ${ }^{1}$, and jinhua ding ${ }^{1}$ \\ ${ }^{1}$ Ningbo Medical Treatment Centre Li Huili Hospital
}

June 16, 2021

\begin{abstract}
Abstract Objectives: This study aimed to evaluate the clinical utility of serum leukocyte counts (SLCs) for initial antibiotic use in Chinese women with mastitis who presented to the emergency department. Materials and Methods: Electronic medical records of breastfeeding women with mastitis were reviewed. Patients were divided into two groups according to the level of SLCs: slight elevated leukocyte counts (SELC, 1.0-1.5*10^9 cells/L); marked elevated leukocyte counts (MELC, >1.5*10^9 cells/L ). Treatment outcomes including rates of treatment failure and breast abscess formation were compared. Results: The rates of treatment failure and breast abscess were $12.7 \%$ and $7.1 \%$ in overall population and $7.7 \%$ and $6.4 \%$ in MELC group, respectively. In SELC group, treatment failures were observed in $29.7 \%$ and $6.4 \%$ patients without or with antibiotics, and the difference was significant $(\mathrm{OR}=4.207,95 \%$ CI 1.318- 13.424$)$; breast abscess was observed in $12.5 \%$ and $2.1 \%$ patients, and the difference was not significant $(\mathrm{OR}=6.571,95 \%$ CI 0.793-54.481). Mean time to normal appearance of the breast or normal temperature was shorter in patients with antibiotics $(3.8 \pm 1.7$ and $4.3 \pm 2.1$ days) than in patients without antibiotic use (2.5 \pm 1.1 and $3.0 \pm 1.3$ days $), \mathrm{p}<0.001$. Conclusion: Our study found that there were better clinical outcomes in patients with SELC or MELC when antibiotic was initially administrated compared to those without antibiotics, whicha indicates that SLC is an easy and practical reference index for gudiing antibiotic use, and patients who have an elevated SLC should be treated with antibiotic. Key Words: mastitis, antibiotic, serum leukocyte count
\end{abstract}

\section{INTRODUCTION}

Acute mastitis is a common problem during breastfeeding, and it is estimated that approximately one in five breastfeeding women has experienced acute mastitis. ${ }^{1-3}$ Mastitis episodes are often characterized by rapid progression with local redness and breast pain and are accompanied by systemic symptoms, including fever, headache, nausea, vomiting, etc. Unlike mastitis sufferers abroad who get medical help from community, mastitis sufferers in mainland China often receive medical help from physicians in the hospital, and most of them have to go to the emergency department (ED) because of high fever, intolerable pain and fear of breastfeeding cessation.

The underlying causes of acute mastitis include stress, over nutrition, overreliance on breast pumps, and a lack of breastfeeding experience. Two direct causes of acute mastitis, milk stasis and bacterial infection, are important and determine the treatment strategies. ${ }^{4,5}$ Conservative treatments, the purpose of which is to relieve milk stasis and that include hot or cold compresses, acupuncture and the application of cabbage leaves, have been demonstrated to be inconclusive for managing milk stasis. ${ }^{6}$ Numeroud literature showed that therapeutic breast massage was helpful for emptying the breast and relieving milk stasis. ${ }^{7-9}$ Antibiotic targeting pathogenic organism is theoretically useful for eliminating inflammation and decreasing abscess formation. However, in some situations, acute mastitis is just an inflammatory condition, and there is no pathogenic bacterial infection. Therefore, conservative treatments seem adequate, and the additional use of antibiotics may be unnecessary in such situations. 
In practice, although the role of conservative treatments for relieving milk stasis is inconclusive, the following treatments are still important for acute mastitis: breast massage for emptying the breast, ibuprofen for analgesia, a hot compress applied prior to breastfeeding for stimulating milk flow, and a cool compress applied after breastfeeding for reducing swelling and pain. In addition, oral or intravenous flucloxacillin is preferred for mastitis episodes that need antibiotics, and clindamycin is chosen for the patient who is allergic to penicillin. If the signs and symptoms improve within 24-48 hours in patients who are prescribed antibiotics, a total duration of five to seven days is encouraged; if the signs and symptoms are not improved or are even worse, antibiotics with different mechanisms are started immediately, and human milk is collected for cultivation and sensitivity tests. ${ }^{4}$

Two inflammatory markers, serum leukocyte counts (SLCs) and C-reactive protein (CRP), are widely used in acute inflammatory diseases, such as acute appendicitis and cholecystitis. However, the associations between these two inflammatory markers and infectious diseases are complex, and few studies focusing on these two inflammatory markers and acute mastitis have been reported. Osterman $\mathrm{K}$ et al. conducted a prospective study to compare serum SLCs and CRP among 41 episodes of lactation mastitis. ${ }^{10}$ Bacterial cultivation of breast milk was performed in all patients, and patients were classified into two groups based on the presence of potentially pathogenic bacteria: group A without potentially pathogenic bacteria and group B with potentially pathogenic bacteria. They found that the mean SLCs were significantly higher in group B than in group A; however, although the levels of CRP in both groups were elevated, there was no significant difference between the two groups. ${ }^{10}$

A positive relationship between higher SLCs and the presence of potentially pathogenic bacteria indicates that mastitis with higher SLCs is more likely to be infectious disease and should be treated with the addition of antibiotics as well as conservative treatments. However, no relative study has been performed since then. Therefore, the purpose of our study was to describe the clinical characteristics, treatment regimens and treatment outcomes of acute mastitis in breastfeeding women and to further explore the value of SLCs for initial antibiotic use in breastfeeding women with acute mastitis in mainland China.

\section{MATERIAL AND METHODS}

\subsection{Study population}

The study included breastfeeding women with acute mastitis who were treated in Ningbo Medical Center Lihuili Hospital between January 2016 and August 2018. The inclusion criteria were the followings: (1) every patient was diagnosed with acute mastitis according to the widely accepted criteria of mastitis (see below); (2) blood sample for SLCs was routinely obtained in all patients directly or shortly after presentation to the $\mathrm{ED}$, and if there was any suspicion of breast abscess, diagnostic breast ultrasound was performed; (3) conservative treatments including breast massage for emptying the breast, ibuprofen for analgesia, a hot compress applied prior to breastfeeding for stimulating milk flow, and a cool compress applied after breastfeeding for reducing swelling and pain, were performed on every patient; (4) common breast signs, including inflammatory characteristics of redness, swelling, heat and pain in the breast and systemic symptoms, including fever, chill, nausea and vomiting, were documented in medical records; (5) follow-up data were necessary, including the name of the antibiotic, the duration of the antibiotic, and the treatment outcome. If women attended the hospital for more than one episode of mastitis, we included only the first admission. The exclusion criteria included the followings: (1) nonpostpartum mastitis; (2) special breast inflammation, including plasma cell mastitis (PCM), granulomatous lobular mastitis (GLM) and breast tuberculosis; (3) the presence of breast abscess before treatment; (4) inflammatory breast carcinoma (IBC) with similar breast signs to acute mastitis; and (5) having received medicinal management for current mastitis before visiting the emergency department. The principles outlined in the Declaration of Helsinki were followed,and written informed consent was obtained from every patient.

\subsection{Definitions}

The definition of mastitis was adopted with widely accepted criteria: the presence of inflammatory characteristics including localized redness, swelling, heat and pain in the breast accompanied by a temperature of 
$38.5^{\circ} \mathrm{C}$ or greater, chills, flu-like aches, and systemic illness. ${ }^{11}$ Treatment outcomes were extracted from the medical records. Treatment failures were determined when there were any of the following situations: (1) deterioration of signs or symptoms; (2) an emerging breast abscess; or (3) switching to a new antibiotic or changing the initial antibiotic. SLCs from routine blood tests were collected at first presentation, and patients were classified into three groups based on the levels of leukocyte counts: 31 patients had a normal leukocyte count (NLC, $<1.0 \times 10^{\wedge} 9$ cells/L); 111 patients had a slightly elevated leukocyte count (SELC, 1.0-1.5×10^9 cells/L); and 156 patients had a markedly elevated leukocyte count (MELC, $>1.5 \times 10^{\wedge} 9$ cells/L). Patients were further divided into subgroups according to the use of antibiotics. The number of patients in the NLC group was small; therefore, in the current study, we explored the value of SLCs in patients with elevated leukocyte counts, including patients with SELCs and MELCs.

\subsection{Data collection}

Electronic medical records were identified and the following data elements were derived: age, postpartum time, parity, history of mastitis, time from onset, laterality, pain, swelling, heat and redness in the breast, breast mass, body temperature and SLCs. Antibiotic use and clinical outcomes were also collected from medical records.

Patients were asked to return to the hospital for follow-up on the seventh day and to report the current situation by telephone if they could not return to the hospital for any reason. For patients who returned to the hospital, a clinical physician could make an efficacy evaluation by comparing the signs and symptoms between their first presentation and the last follow up; for patients who did not return to the hospital, an efficacy evaluation was made by the patients themselves and was reported to the physicians by telephone.

\subsection{Statistical analysis}

The Chi-square test or Fisher's exact test was used to analyze categorical variables, and t-test was used to analyze continuous variables. All statistical tests were two-sided, and a p value of less than 0.05 indicated statistical significance. The odds ratio (OR) and $95 \%$ confidence interval (CI) were also calculated. Statistical analyses were performed with SPSS 20.0 software (SPSS, Chicago, IL, http:// www. spss.com).

\section{RESULTS}

During study period, a total of 418 women were admitted to emergency department with a definite diagnosis of mastitis, finally, 267 patients were eligible for this study (Figure 1 ). The mean ( \pm SD) age of the included patients was $27.5( \pm 2.40)$ years, with a range from 20 to 36 years. At presentation, $252(94.4 \%)$ had breast redness, $234(87.6 \%)$ had breast swelling, $251(94.0 \%)$ had breast heat, $260(97.4 \%)$ had severe pain in the breast, and $228(85.4 \%)$ reported breast nodules/lumps. The characteristics of patients in the SELC and MELC groups are shown in Table 1 .

Antibiotics were prescribed to $200(74.9 \%)$ patients, including 38 (14.2\%) patients prescribed oral antibiotics and $162(60.7 \%)$ patients prescribed intravenous antibiotics. Initial use of flucloxacillin, cephradine and clindamycin occurred in $186(69.7 \%), 6(2.2 \%)$ and $8(3.0 \%)$ patients, respectively. The rates of initial antibiotic use in patients with SELCs and MELCs were $42.3 \%$ and $98.1 \%$, respectively, $\mathrm{p}<0.001$ (Table 2).

There were 33 (12.4\%), 53 (19.8\%) and $13(5.2 \%)$ patients who did not perform therapeutic breast massage, who stopped breastfeeding or both, respectively; their counterparts consisted of 11 (9.9\%), 25 (22.5\%), and $6(5.4 \%)$ patients with SELCs and 22 (14.1\%), 28 (17.9\%), and 7 (4.5\%) patients with MELCs, respectively.

Among all 267 eligible patients, $230(86.1 \%)$ patients returned to the hospital for review before or at the seventh day after initial treatment, and another 37 (13.9\%) patients did not provide treatment outcomes by telephone.

The rate of treatment failure was $12.7 \%(34 / 267)$ in the overall population and $7.7 \%(12 / 156)$ in the MELC group. In the MELC group, only three patients did not receive an initial antibiotic but received subsequent antibiotics due to uncontrolled symptoms, and one of these patients developed a breast abscess. Of the remaining 153 patients, 9 patients developed breast abscesses. 
The rate of treatment failure was 19.8\% (22/111) in the SELC group, and treatment failures included 19 patients who were switched to antibiotic and 3 patients who had a change of their initial antibiotic. In 47 patients with initial antibiotic use, the rate of treatment failure was $6.4 \%(3 / 47)$, and in 64 patients without initial antibiotic use, the rate of treatment failure was $29.7 \%(19 / 64)$. The difference in patients with or without initial antibiotic use was significant ( $\mathrm{OR}=4.207,95 \%$ CI 1.318-13.424) (Table 3) .

The overall rate of breast abscess was $7.1 \%$ (19/267). The proportion was similar in each group: 8.1\% (9/111) in the SELC group and 6.4\% (10/156) in the MELC group. In the SELC group, the proportions were $2.1 \%$ $(1 / 47)$ and $12.5 \%(8 / 64)$ when antibiotics were initially used or not used, respectively; the difference was not significant but showed an obvious trend $(\mathrm{OR}=6.571,95 \%$ CI 0.793-54.481) (Table 3). All the breast abscesses were managed with repeat US-guided aspiration, and $84.2 \%(16 / 19)$ achieved complete resolution, while $15.8 \%(3 / 19)$ resorted to surgical drainage.

In the SELC group, the mean time until normal appearance of the breast and normal temperature was significantly shorter in patients with antibiotic use $(3.8 \pm 1.7$ and $4.3 \pm 2.1$ days $)$ than in those without antibiotic use $(2.5 \pm 1.1$ and $3.0 \pm 1.3$ days), $\mathrm{p}<0.001$ (Table 4$)$.

\section{DISCUSSION}

The World Health Organization has suggested that breast milk culture and sensitivity testing should not be routinely performed and should be performed if there is any of the following situations: (1) no response or poor response to the antibiotic within 2 days; (2) the mastitis recurs; (3) hospital-acquired mastitis; (4) allergic to the usual therapeutic antibiotic; and (5) in severe or unusual cases. Therefore, in clinical practice, antibiotic use for acute mastitis has always been empirical worldwide, and even in developed countries such as Australia, ${ }^{1,12}$ Spain $^{13}$ and the USA, ${ }^{14,15}$ as high as $77-97 \%$ patients with breastfeeding mastitis are prescribed wih toral or intravenous antibiotics without direct evidence of bacterial infection. Indeed, in China mainland, antibiotic use is usually based on the level of SLCs and the severity of symptoms and signs, but not on the results of bacteriological analyses, although the latter has been helpful in determining the presence of pathogenic bacteria.

A previous study found that higher SLCs were associated with the existence of potentially pathogenic bacteria. ${ }^{10}$ Higher SLCs may indicate a higher chance of infection, which is useful in certain clinical conditions. For patient who is unwilling to undergo bacterial cultivation of breast milk or in the most of medical agencies where bacterial cultivation is not a routine practice, antibiotic use based on the SLCs seems to be a practical choice. SLC is routinely obtained in the work-up of patients with acute mastitis in China. If SLC is above the normal level, antibiotics are often recommended by attending physicians in the emergency department; otherwise, antibiotics are not advised. To the best of our knowledge, the study is the first to explore the value of SLCs for initial antibiotic use in breastfeeding mastitis.

In our study, the majority of $(98.1 \%)$ patients with MELCs were prescribed an initial antibiotic, so we could not assess the necessity of initial antibiotic use effectively. However, we found a great difference in patients with MELCs; $5.5 \%$ (9/153) versus 100.0\% (3/3) patients with MELCs had a disease progression when the antibiotic was or not given at their first presentation to the hospital. The result indicates that initial antibiotic use is still essential for acute mastitis with MELCs.

Our study also showed that there was a better clinical outcome in patients with SELCs when antibiotic was given initially. There should be two possibilities for mastitis: the presence of both inflammation and infection in the breast, or the presence of only inflammation but no infection in the breast. However, we could not distinguish these two situations clearly without the evidence of bacterial cultivation. Even so, for patients with SELCs, there was an absolute difference of $23.3 \%$ on the rate of treatment failure between patients with antibiotic use and without antibiotic use, which demonstrated that appropriate antibiotics should also be recommended for patients with SELCs.

In the current study, the rate of breast abscess was $7.1 \%$, which was higher than that in the large Australian study which found that $3 \%$ of breastfeeding women with mastitis developed breast abscess. ${ }^{16}$ The difference 
in race and characteristics of patients in two studied may contribute to the different rate of breast abscess.

The limitations also should be acknowledged. First and most important, the present study was a singleinstitution, observational, retrospective study, which decreases the external validity of our work. Second, the level of SLCs in acute mastitis was always elevated, and acute mastitis patients with normal SLCs in clinical practice were rare; thus, the present study did not include such patients. Thus, the value of SLCs for antibiotic use in such patients could not be explored in the study. Third, in our study, milk cultivation and bacteriological analysis were not routinely performed, and antibiotics were initially used to target $S$. aureus , which was found to be the most pathogenic bacteria in previous studies. ${ }^{17-20}$

In this study, we found that there was a better clinical outcome for patients with SELCs when antibiotic was given compared to when an antibiotic was not given. In addition, there was a good clinical outcome for patients with MELCs when antibiotic was given initially. These results suggest that, in China mainland, SLCs may have a reference value for antibiotic use in breastfeeding women with acute mastitis, and those sufferering from acute mastitis with elevated SLCs should be prescribed with antibiotic their first presentation to hospital. Of course, large-scaled, randomized controlled trials (RCTs) of higher quality are still required to confirm our findings.

\section{ACKNOWLEDGEMENTS}

The authors sincerely thank Dr. Jiahui Yang for assistance in data collection, and Drs. Yixia Zhou and Ye Zhu for their invaluable guidance in proofreading this article.

\section{DISCLOSURE}

All authors declare that there is no conflict of interest.

\section{FUNDING SUPPORT}

The work is funded by the Natural Science Foundation of Ningbo, Grant Number is 2018A610325.

\section{DATA AVAILABILITY STATEMENT}

The data that support the findings of this study are available from the corresponding author upon reasonable request.

\section{REFERENCES}

Amir LH, Forster DA, Lumley J, et al. A descriptive study of mastitis in Australian breastfeeding women: incidence and determinants. BMC Public Health. 2007; 7: 62.

2. Kinlay JR, O'Connell DL, Kinlay S. Incidence of mastitis in breastfeeding women

duringthe six months after delivery: a prospective cohort study.Med $J$ Aus. 1998; 169:

310-312.

3. Thompson JF, Roberts CL, Currie M, Ellwood DA: Prevalence and persistence of health problems after childbirth: associations with parity and method of birth.Birth. 2002; 29:83-

94.

4. Amir LH, Academy of breastfeeding Medicine Protocol Committee: ABM clinical protocol \#4: Mastitis, revised March 2014. Breastfeed Med. 2014; 9: 239-243.

5. Dixon JM, Khan LR. Treatment of breast infection. BMJ. 2011; 342: d396.

6. Mangesi L, Zakarija-Grkovic I. Treatments for breast engorgement during lactation.

Cochrane Database Syst Rev. 2016; 6:CD006946. 
7. Bolman M, Saju L, Oganesyan K, et al. Recapturing the art of therapeutic breast massage during breastfeeding. JHum Lact. 2013; 29: 328-331.

8. Zhao C, Tang R, Wang J, et al. Six-Step Recanalization Manual Therapy: A Novel Method for Treating Plugged Ducts in Lactating Women. J Hum Lact. 2014; 30: 324-330.

Witt AM, Bolman M, Kredit S,] et al. Therapeutic Breast Massage in Lactation for the Management of Engorgement, Plugged Ducts, and Mastitis. J Hum Lact. 2016; 32: 123-131.

Osterman K, Rahm VA. Lactation Mastitis: Bacterial Cultivation of Breast Milk, Symptoms, Treatment, and Outcome. J Hum Lact. 2000; 16: 297-302. 11. Lawrence RA. The puerperium, breastfeeding, and breast milk.Curr Opin Obstet Gynecol. 1990; 2: 23-30. 12. Fetherston C. Management of lactation mastitis in a Western Australian cohort. Breastfeed

Rev. 1997; 5: 13-19.

13. Mediano P, Fernández L, Rodríguez JM, et al. Case-control study of risk factors for infectious mastitis in Spanish breastfeeding women. BMC Pregnancy Childbirth. 2014;14 195.

Foxman B, D'Arcy H, Gillespie B, et al. Lactation mastitis: occurrence and medical management among 946 breastfeeding women in the United States.Am J Epidemiol.

2002; 155: 103-114.

15. Wambach KA. Lactation mastitis: a descriptive study of the experience. J Hum Lactc. 2003;

19: 24-34.

16. Amir LH, Forster D, McLachlan H, et al. Incidence of breast abscess in lactating women: report from an Australian cohort. BJOG. 2004; 111:1378-1381.

17. Kvist LJ, Larsson BW, Hall-Lord ML,et al. The role of bacterial in lactational mastitis and some considerations of the use of antibiotic treatment. Int Breastfeed J. 2008; 3: 6.

18. Osterman KL, Rahm VA. Lactation mastitis: bacterial cultivation of breast milk, symptoms, treatment and outcome. J Hum Lact. 2000; 16: 297-302.

19. Farahnik B, Murase JE. Antibiotic safety considerations in Methicillin-resistant Staphylococcus aureus postpartum mastitis. J Am Acad Dermatol.2016; 75: e149.

20. Hagiya H, Shiota S, Sugiyama W, et al. Postpartum breast abscess causedby communityacquired methicillin-resistant Staphylococcus aureus in Japan.Breastfeed Med. 2014; 9:

45-46.

\section{FIGURE LEGEND}

Fig 1. Flow-chart of the cohort selection for the study. 


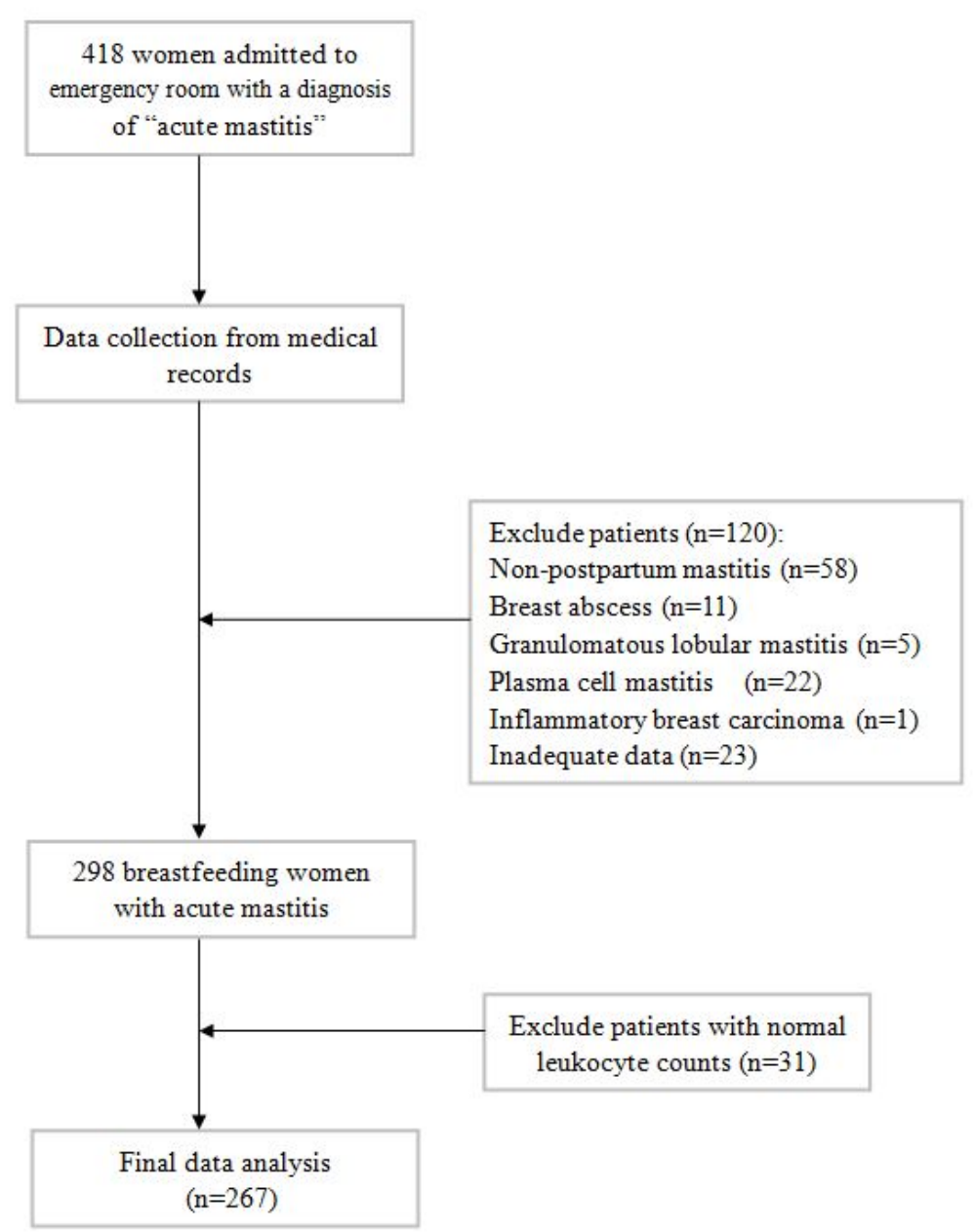

\section{Hosted file}

Table 1.docx available at https://authorea.com/users/420126/articles/526499-the-clinialutility-of-serum-leukocyte-counts-for-initial-antibiotic-use-in-acute-mastitis-theexperience-from-china

\section{Hosted file}

Table 2.docx available at https://authorea.com/users/420126/articles/526499-the-clinialutility-of-serum-leukocyte-counts-for-initial-antibiotic-use-in-acute-mastitis-theexperience-from-china

\section{Hosted file}

Table 3.docx available at https://authorea.com/users/420126/articles/526499-the-clinialutility-of-serum-leukocyte-counts-for-initial-antibiotic-use-in-acute-mastitis-theexperience-from-china

\section{Hosted file}


Table 4.docx available at https://authorea.com/users/420126/articles/526499-the-clinialutility-of-serum-leukocyte-counts-for-initial-antibiotic-use-in-acute-mastitis-theexperience-from-china 